\title{
Chronische Rückenbeschwerden
}

\section{Schmerzreduktion durch Opioide klinisch nicht relevant}

— Selbst mit höher dosierten Opioidanalgetika lässt sich bei chronischem Rückenschmerz keine klinisch relevante Linderung der Beschwerden erreichen. Dies ist das Ergebnis einer Metaanalyse, in der Wirksamkeit, Verträglichkeit und dosisabhängige Effekte von Opioiden untersucht wurden. In einem systematischen Review verschafften sich Christina Abdel Shaheed und Kollegen von der Universität Sydney einen Überblick über die placebokontrollierten randomisierten Studien zum Thema. Insgesamt wurden 20 Studien mit 7.925 chronischen Rückenschmerzpatienten, die über maximal zwölf Wochen mit Opioiden behandelt worden waren, analysiert [Shaheed et al. JAMA Intern Med. online 2016 May 23]. Primärer Bewertungsmaßstab war der Schmerz, dessen Stärke auf einer Skala von 0-100 gemessen wurde. Eine Reduktion von mehr als 20 Score-Punkten galt dabei als klinisch relevant. Es ergab sich insgesamt eine mäßiggradige Evidenz dafür, dass
Opioidanalgetika chronische Rückenschmerzen kurz- bis mittelfristig lindern können. Innerhalb eines Bereichs von 40-240 mg Morphinäquivalenten/Tag konnte zwar mit steigender Dosis eine stärkere Schmerzreduktion beobachtet werden, eine klinisch relevante Verbesserung von mehr als 20 Score-Punkten wurde aber auch mit der höchsten Dosierung nicht erreicht. Hoch war jedoch die Aussteigerquote aus den Studien: Infolge der Nebenwirkungen oder wegen mangelnder Wirksamkeit hatte mindestens jeder zweite Studienteilnehmer die Therapie abgebrochen. Für Patienten mit chronischen Rückenschmerzen, die Opioidanalgetika vertragen, könnten diese kurzfristig eine geringe Schmerzreduktion herbeiführen. Der erreichte Effekt sei für die innerhalb der Leitlinien empfohlenen Dosierungen allerdings klinisch wahrscheinlich nicht relevant, so Abdel Shaheed und Kollegen.

Dr. Christine Starostzik

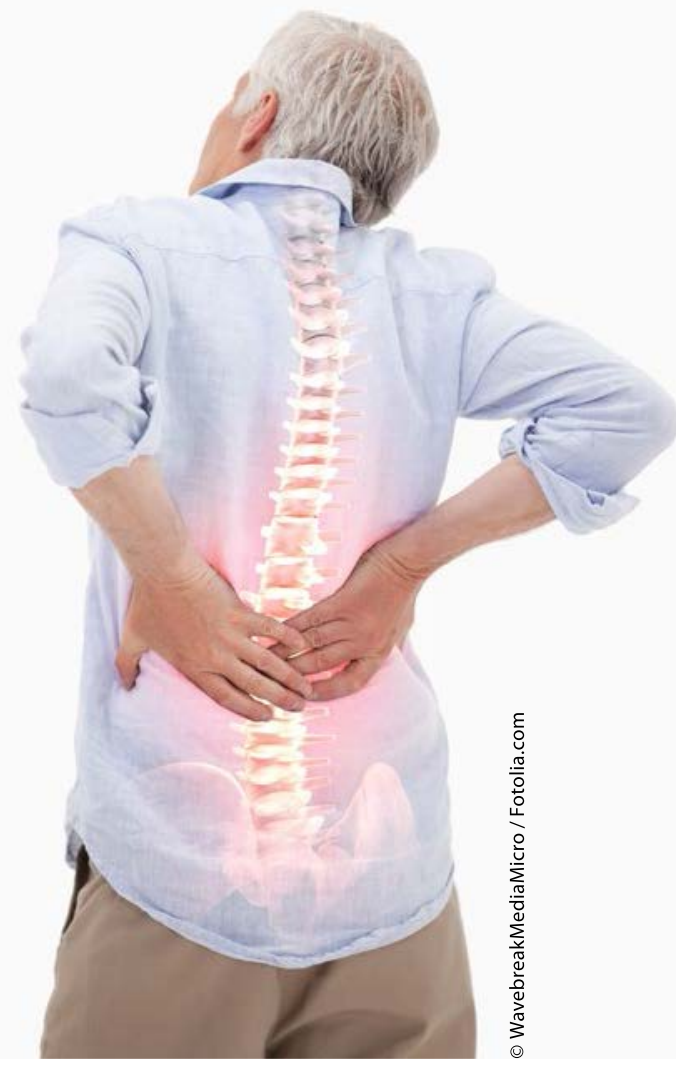

Nutzen bei Allodynie

\section{Botulinumtoxin lindert neuropathische Schmerzen}

— Periphere neuropathische Schmerzen lassen sich durch subkutane Injektionen von Botulinumtoxin (BTX) A bessern. Vor allem Patienten mit Allodynie und geringem thermalem Defizit profitieren. Experimentelle Untersuchungen der vergan- genen Jahre haben gezeigt, das BTX A über seine Effekte auf den Muskeltonus hinaus auch analgetisch wirkt. Ein Team um Nadine Attal vom Institut national de la santé et de la recherche médicale (INSERM) in Boulogne-Billancourt, Frankreich, hat in einer kleinen, doppelblinden und placebokontrollierten Studie untersucht, ob BTX A auch Vorteile für die Behandlung von Patienten mit Neuropathieschmerzen mit sich bringt [Attal et al. Lancet Neurol online 2016
Feb 29]. Vor den Injektionen hatte der von den Patienten berichtete Schmerzgrad auf einer Skala von 0 (keine Schmerzen) bis 10 (maximal vorstellbarer Schmerz) in der BTX-A-Gruppe bei 6,5 Punkten gelegen - ein Wert, der starke Schmerzen bedeutet. Nach 24 Wochen, also zwölf Wochen nach der zweiten Injektionsrunde, lag der Punktwert bei 4,6, was mittelstarke Schmerzen bezeichnet. In der Placebogruppe war ein Ausgangswert von 6,4 Punkten gemessen worden, nach 24 Wochen waren es 5,8 Punkte. Statistisch bedeutsam war die Differenz bei $30 \%$ iger Schmerzreduktion Reduktion (65\% vs. $25 \%)$.

Neuropathiepatienten mit Allodynie und einer wenig beeinträchtigten Temperaturwahrnehmung sprachen im Durchschnitt besser an als Patienten ohne diese Merkmale. Dies korrelierte damit, dass Responder in Hautbiopsien eine höhere Dichte an intraepidermalen Nervenfasern aufwiesen.

Robert Bublak 